\title{
CCR2/CCR5 Antagonist BMS-813160
}

National Cancer Institute

\section{Source}

National Cancer Institute. CCR2/CCR5 Antagonist BMS-813160. NCI Thesaurus. Code C139550.

An antag onist of both human C-C chemokine receptor types 2 (CCR2; CD192) and 5 (CCR5; CD195), with potential immunomodulating and antineoplastic activities. Upon administration, CCR2/CCR5 antagonist BMS-813160 specifically binds and prevents the activation of both CCR2 and CCR5. This inhibits the activation of CCR2/CCR5-mediated signal transduction pathways and may inhibit inflammatory processes, angiogenesis, tumor cell migration, tumor cell proliferation and invasion. The G-protein coupled chemokine receptors CCR2 and CCR5 are expressed on the surface of monocytes and macrophages, and stimulate their migration and infiltration; they play key roles in inflammation and autoimmune disease. CCR2 and CCR5 are overexpressed in certain cancer cell types, and are also involved in angiogenesis, and in tumor cell migration, proliferation and metastasis. 